\title{
POSIBLES EFECTOS SOCIOECONÓMICOS DE LA EFICIENCIA ENERGÉTICA DE LOS NUEVOS SISTEMAS DE ILUMINACIÓN BASADOS EN TECNOLOGÍA LED
}

\author{
Socio-economic potential effects of energy efficiency \\ of new lighting systems based in led technology \\ Carlos VelásqueZ ${ }^{1}$ y Francisco Espín ${ }^{2}$
}

\begin{abstract}
Cómo citar: Velásquez, C., \& Espín, F. (2018). Posibles efectos socioeconómicos de la eficiencia energética de los nuevos sistemas de iluminación basados en tecnología LED. Ciencia, Ingenierías y Aplicaciones, 1(1), 9-22. doi:http://dx.doi.org/10.22206/ cyap.2018.v1i1.pp9-22
\end{abstract}

\section{Resumen}

Este trabajo presenta una exposición de posibles efectos socioeconómicos que pueden presentarse con la eficiencia energética de los nuevos sistemas de iluminación basados en tecnología LED en su incursión masiva en algunos países de América del Sur y América Central. El documento está orientado a presentar datos y resultados técnicos y cientificos del estudio de los sistemas de iluminación LED con las correspondientes consecuencias positivas y negativas del ambiente social y económico de un país. Se ha llevado a cabo una investigación documental y se ha obtenido como resultado un análisis de posibles escenarios a tener en cuenta por las instituciones encargadas de la adquisición masiva de equipos para la iluminación exterior. Se ha analizado los espectros de las fuentes de luz y la demanda visual de los usuarios desde una perspectiva social.

Palabras clave: Iluminación exterior; eficiencia energética; espectros; demandas visuales.

\footnotetext{
1 Escuela Politécnica Nacional. Ecuador. Correo electrónico: carlos.velasquez@iner.gob.ec

2 Escuela Politécnica Nacional. Ecuador.
} 


\begin{abstract}
This paper presents an exhibition of the potential socio-economic effects that may occur with the energy efficiency of new lighting systems based on LED technology in its massive incursion in some countries of South America and Central America. The document is aimed at presenting data and scientific and technical results of the study of LED lighting systems with its corresponding positive and negative consequences in social and economic environment of a country. It has carried out a documentary research, and has been obtained as a result an analysis of possible scenarios to be considered by the institutions responsible for the massive purchase of equipment for outdoor lighting. It was analysed the spectra of the light sources and the visual requirements of users from a social perspective.
\end{abstract}

Keywords: Exterior lighting, energy efficiency, spectra, visual requirements. 


\section{Introducción}

La tecnología tradicional para la iluminación vial ha estado dominada en las últimas décadas por lámparas y luminarias de diversas clases como sodio de alta presión, sodio de baja presión y vapor de mercurio (Boyce, 2009). El costo por suministro de energía debido al empleo de este tipo de fuentes luminosas representa una parte importante del presupuesto de municipios, empresas distribuidoras de energía, distritos o la entidad correspondiente que se encuentre a cargo de la iluminación vial.

La luz de estado sólido (solid state light, SSL) y en especial los diodos emisores de luz (light-emitting diode, LED), han encontrado una aplicación industrial en la construcción de luminarias LED para un alumbrado vial (Medina, Avrenli, \& Benekohal, 2013) "más eficiente". América del Norte y Europa son las regiones que tienen mayor implementación de esta nueva tecnología (Valentová, Quicheron, \& Bertoldi, 2014). El compromiso para detener el cambio climático global y las dificultades económicas para abastecer de suficiente energía eléctrica a algunos países de las Américas del Sur y Central han permitido que sus respectivos gobiernos impulsen políticas destinadas a la eficiencia energética y, en este punto, la iluminación LED ha ingresado con fuerza a la región. No obstante estudios recientes empiezan a reportar graves afectaciones a poblaciones de determinadas especies animales y vegetales que desembocan en fallas en la autoregulación de los ecosistemas (Hecht, 2015).

La transición de tecnología en el alumbrado público es una política seductora para varias administraciones, sin embargo, el enfoque único en la eficiencia energética provoca la pérdida de perspectiva sobre los amplios efectos socio-económicos colaterales que traerá dicho cambio. Lo dicho justifica la generación de un debate y profundizar en el análisis de varios puntos relativamente beneficiosos y perjudiciales de la avalancha LED fundamentados en datos y artículos científicos de 
relevancia ponderativa para el estado de la técnica. El presente trabajo describe algunas de las coyunturas abiertas por la nueva tecnología de los sistemas de iluminación LED en alumbrado exterior para atender la incursión masiva en países como Ecuador, Colombia, Venezuela, Panamá, Costa Rica e Islas del Caribe.

\section{Una perspectiva de ahorro económico}

\section{y beneficio social con la eficiencia energética}

\subsection{Aspectos técnicos para la optimización de energía en alumbrado exterior}

Las luminarias LED tienen diversos parámetros para la maximización de la eficiencia energética en los sistemas de iluminación (DiLaura, Houser, Mistrick, \& Steffy, 2011). Las normativas de cada país garantizan los mínimos niveles de iluminación que deben existir en determinados tipos de vías. Los lentes y el diseño de las posiciones de los LED en una luminaria son capaces de generar nuevas matrices de distribución de intensidades. Los diseños LED con sus respectivos parámetros pueden reemplazar a la tecnología tradicional para cumplir con los criterios de luminancia e iluminancia exigidos.

La vida útil de la luminaria es un eje fundamental, mientras que una lámpara de sodio de alta presión comúnmente empleada en iluminación vial tiene una vida útil entre 28,000 y 32,000 horas según datos reportados por Osram y Phillips (Osram a, 2016) (Philips a, 2016), las luminarias LED, según fabricantes y con un buen diseño mecánico del disipador de calor efectivo puede alcanzar una vida útil de 100,000 horas (Phillips b, 2016) (Osram b, 2012) (Figura 1), sin embargo este valor es obtenido bajo condiciones de laboratorio y es resultado de extrapolaciones por lo que en la práctica esta cifra es cada vez "más conservadora" por parte de los fabricantes. 


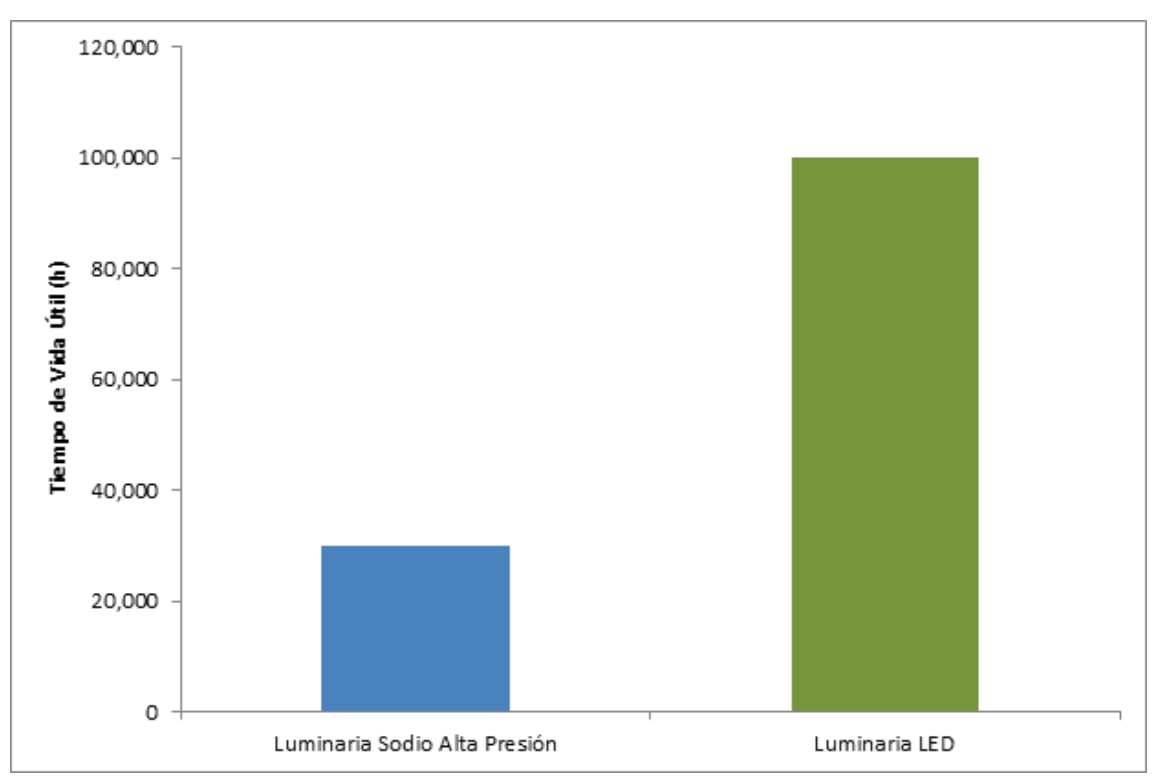

Figura 1. Vida útil de las Luminarias de Sodio de Alta Presión y Luminarias LED

Las propiedades de color en el LED son otro tipo de aporte. El diseño de un sistema de iluminación se presenta dependiendo de la actividad que se realiza en el lugar. Si el objetivo es alumbrar a peatones cuya actividad principal es caminar o a conductores de automóviles, motocicletas que únicamente conducen, simplemente se deben tener en cuenta los niveles de luminancia e iluminancia, más no el espectro de la luz con la que se ilumina.

La mayoría de los niveles exigidos por normativas se encuentran en visión fotópica, sin embargo, no se debe desconocer que la realidad en varios países de América del Sur y América Central, debido a la cultura de los ciudadanos, hace que las vías no tengan un uso específico, es decir actividades que van más allá de las mencionadas anteriormente. 


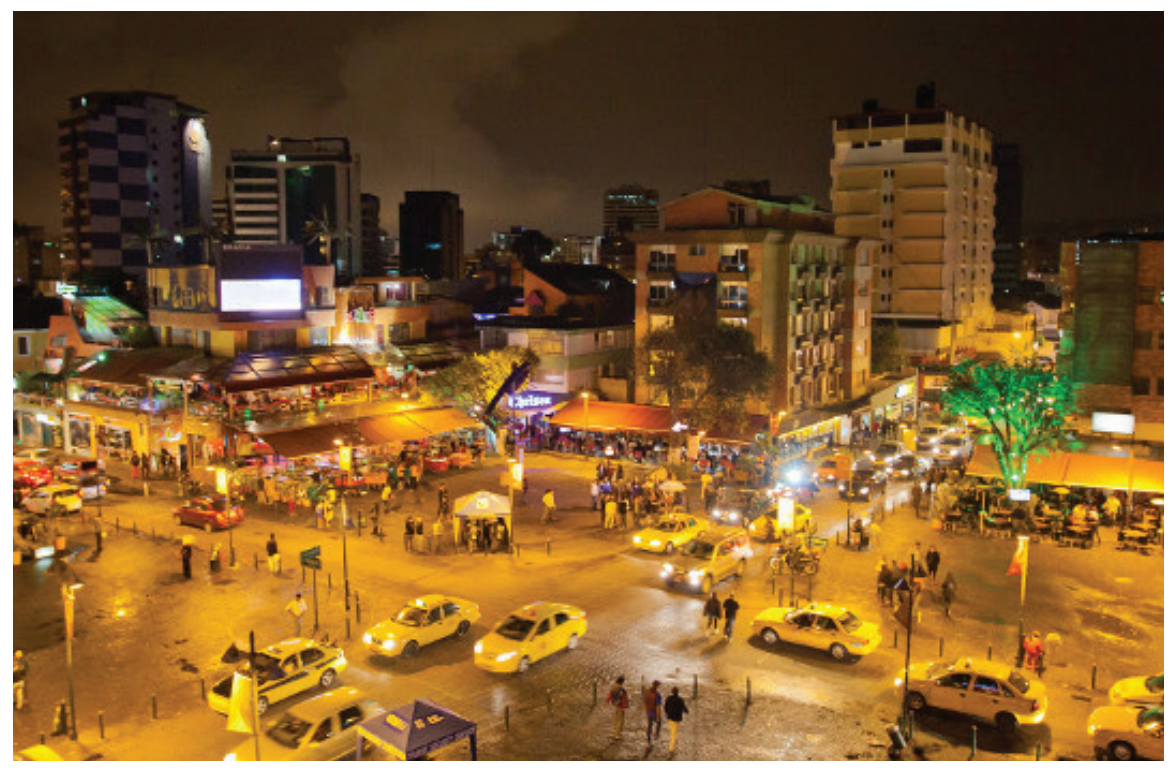

Figura 2. Calle Juan León Mera y Av. Mariscal Foch ubicadas en el centro de la ciudad de Quito, Ecuador.

Las lámparas de sodio de alta presión tienen un espectro con dos líneas definidas en $589 \mathrm{~nm}$ y $589.6 \mathrm{~nm}$ (Flesch, 2006), cerca del pico de máxima sensibilidad de visión del ser humano. Esto provoca que se miren todas las cosas de color amarillento, lo cual es perfectamente aplicable a las tareas elementales de caminar o conducir; sin embargo, existen vías (especialmente en las capitales de comercio como Caracas, Quito, Guayaquil o Cali) en las que se realizan regularmente actividades que necesitan fuentes luminosas con buenas propiedades de reproducción del color: exposiciones artísticas, desfiles estudiantiles, resaltación de fachadas o monumentos, tarimas para presentaciones (en la Figura 2 se muestra un cruce de calles en Quito donde los ciudadanos comúnmente realizan esta clase de actividades). Dos buenas opciones son las lámparas de haluros metálicos y la luz LED, esta última ofrece varios "tipos de blancos" y en su mayoría se utiliza aquellas enriquecidas en los azules. 


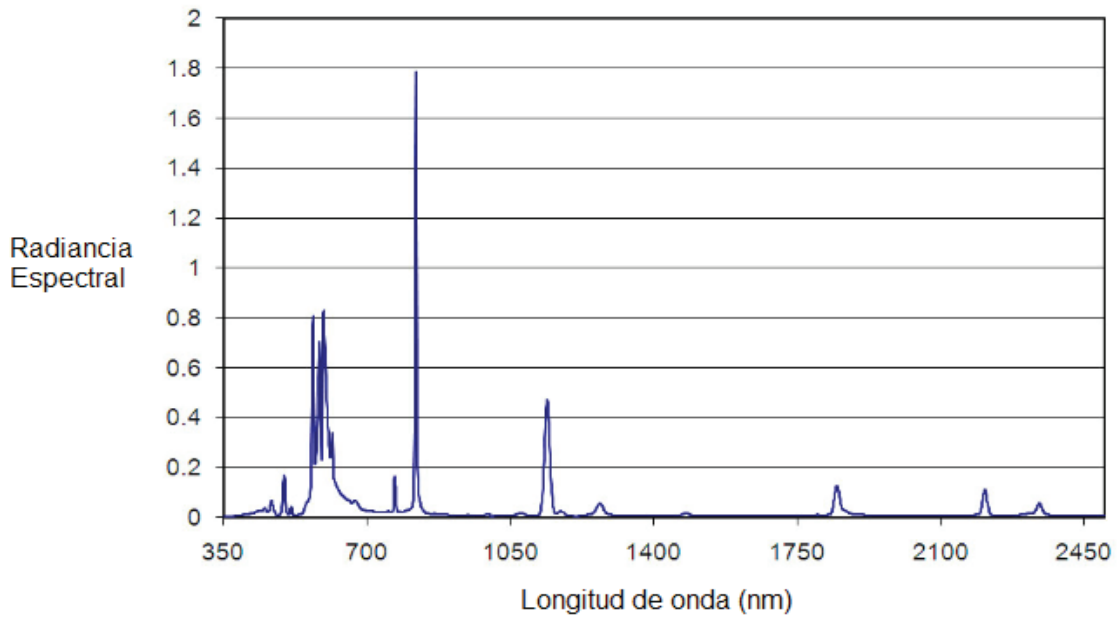

Figura 3. Espectro de una luminaria de descarga de Sodio de Alta Presión de 150W, utilizada actualmente para la iluminación vial en la mayoría de países. Se puede observar los picos de descarga alrededor de $580 \mathrm{~nm}$ (Elvidge, Keith, Tuttle, \& Baugh, 2010).

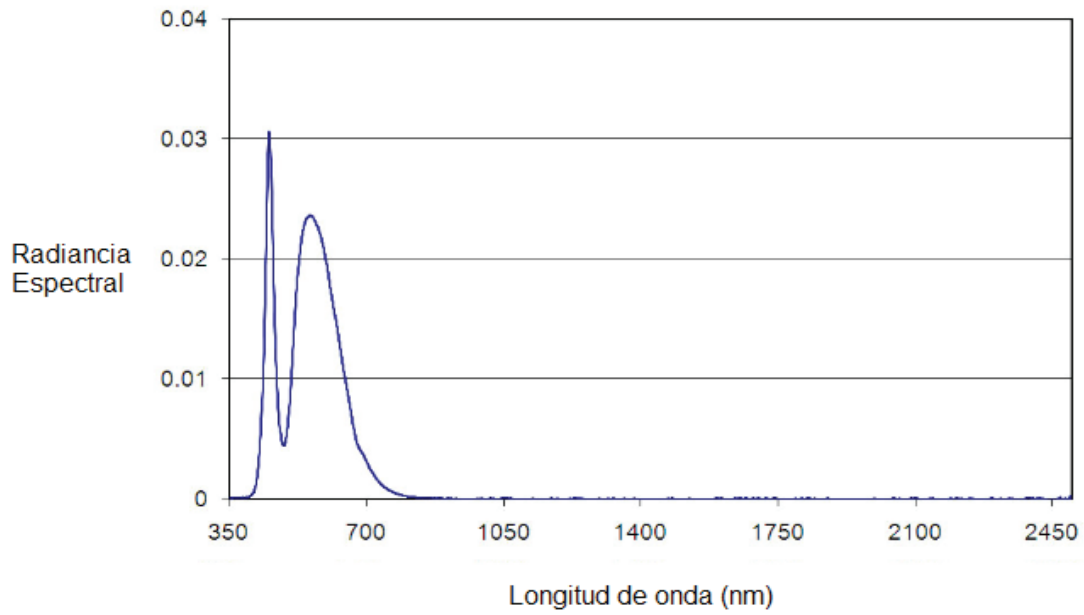

Figura 4. Espectro de una luminaria LED de $125 \mathrm{~W}$, esta muestra una gran radiancia espectral en la zona azul del espectro, actualmente se encuentran reemplazando la iluminación vial (Elvidge, Keith, Tuttle, \& Baugh, 2010).

La dimerización permite controlar la energía consumida por la luminaria, el criterio a emplearse puede ser propuesto en función de la hora a la que se encuentre. Las horas pico deberán funcionar 
utilizando toda su potencia nominal; pero, en la noche avanzada y madrugadas, donde no existe la misma demanda de usuarios se puede reducir la potencia entregada, disminuyendo de esta forma el consumo energético de la ciudad o del país. La definición de los intervalos de tiempo y la potencia necesaria en cada intervalo (un ejemplo propio se muestra en la Figura 5) necesitan un estudio particular que depende de las necesidades de cada ciudad; y puede ser establecido por la empresa encargada de la iluminación vial o exterior.

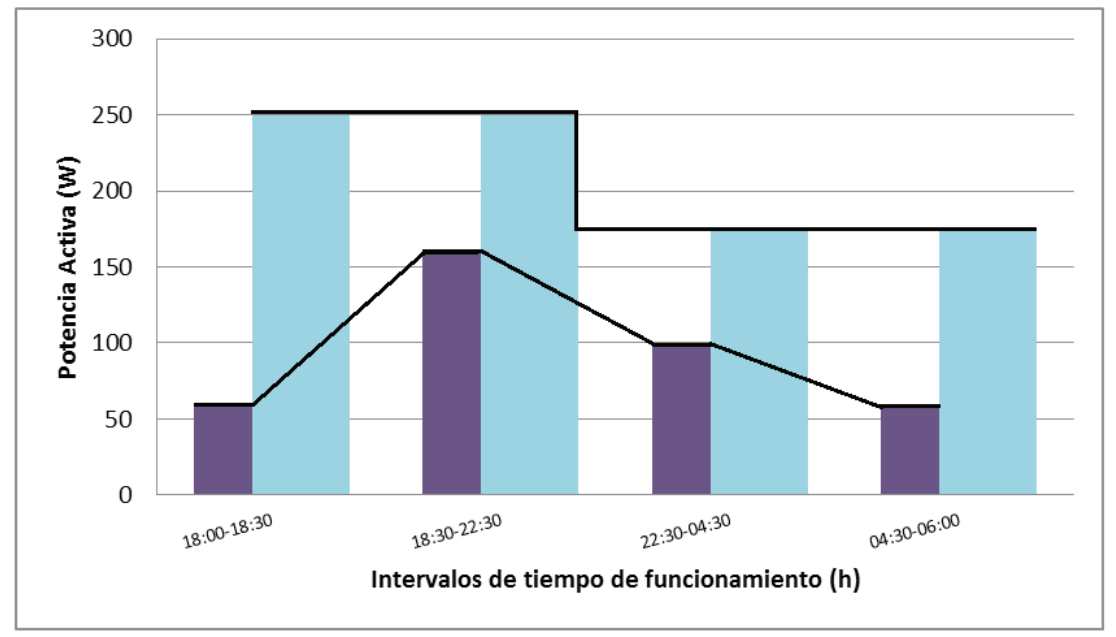

Figura 5. Dimerización de una luminaria led de 160W (barras izquierdas) y una de sodio de alta presión de $250 \mathrm{~W}$ doble nivel de potencia (barras derechas).

\subsection{Visiones de beneficio social a partir de sus ventajas tecnológicas}

El empleo de luz LED en iluminación exterior es relativamente nueva y por medio de los controladores electrónicos se puede garantizar una optimización del consumo de energía que es equivalente a optimizar la rentabilidad de los sistemas de iluminación sin perder los niveles mínimos exigidos por norma.

Los diseños de sistemas de iluminación LED representan una opción que no disminuye el nivel de confort y en determinadas carre- 
teras aumentaría la satisfacción de los ciudadanos que utilizan estos espacios públicos con diversos fines.

Un beneficio más general con el ahorro energético es la reducción de la emisión de dióxido de carbono. El momento de máxima demanda, en muchos países de América del Sur y América Central, se encuentran en funcionamiento centrales térmicas que ayudan a cubrir el pico de demanda energética, de la cual forma parte el alumbrado público. Esta disminución de gas de efecto invernadero es una contribución al mantenimiento del planeta, implica una reducción económica en inversión de eficiencia energética de los países comprometidos y una colaboración social a las medidas contra el calentamiento global.

\section{Una perspectiva de perjuicio social y económico por el deseo de eficiencia energética}

\subsection{Daños medioambientales en ecosistemas pueden afectar al turismo}

La implementación de iluminación LED en islas como Las Galápagos en Ecuador, San Andrés en Colombia, Margarita en Venezuela o cualquier lugar con una biodiversidad abundante en especies raras y turísticas puede influir negativamente en los ecosistemas que son objeto de visita, causando una degradación del lugar y consecuentemente reduciendo los ingresos económicos generados por turismo en los respectivos países.

Los ojos de animales, como los ojos humanos, contienen receptores sensibles a los azules que ayudan a controlar los relojes biológicos y la luz azul excesiva puede confundir al sistema de detección. Esto podría poner en peligro aún más el ciclo de vida ya comprometida de las tortugas marinas en peligro de extinción (Rich \& Longcore, 2006). La evolución se ha encargado de programar a las crías de tortuga para que corran hacia el océano antes de que depredadores costeros 
puedan atraparlas, para hacer este escape, las crías buscan luz de la luna reflejada en el agua (Hecht, 2015).

Las luminarias LED, en especial las enriquecidas en el azul, llevan a tortugas jóvenes en la dirección equivocada ya que muchas veces su temperatura de color correlacionada es cercana a la de la luna, esto empeora si consideramos que la respuesta de foto-orientación de las tortugas en el pico azul de $450 \mathrm{~nm}$ es 10 veces más fuerte que en 600 nm (Hecht, 2015). Se debe considerar también que la dispersión del aire es mucho más fuerte en el azul, añadiendo posibles distracciones (Rich \& Longcore, 2006).

La luz azul también tiene impacto cuando penetra en el mar profundo, hace que otras especies marinas sean vulnerables. Se ha demostrado que afecta a las especies que sincronizan su desove durante la luna llena ya que pueden ser engañadas por el color claro de una falsa luna de LEDs.

Las fuentes con altos contenidos del espectro en los azules atraen polillas y muchos otros insectos, que son una parte vital de los ecosistemas, más que otras fuentes de luz visible (la gente utiliza luces amarillas para evitarlos). Los biólogos han observado que las poblaciones de muchas de las grandes mariposas nocturnas de Europa y Gran Bretaña han caído un 75 por ciento más en los últimos años. Si consideramos estos estudios, la implementación agresiva en América del Sur, rica en insectos, aves y flora especial, se vería gravemente afectada.

En 2014 Ecuador registró ingresos por turismo de 1,487.2 millones de dólares (Ministerio de Turismo, 2014) y Colombia de 4,890 millones de dólares (Minicomercio Industria y Turismo, 2016). Los beneficiarios principales son los habitantes de las zonas turísticas donde la explotación de la belleza natural mediante su exposición es su medio 
diario de supervivencia económica. Un cambio de iluminación con el objetivo de hacer al alumbrado público más eficiente puede converger en la degeneración de los sectores turísticos perdiendo los ingresos económicos y llevando a las ciudades más beneficiadas a un tambaleo económico.

\subsection{Inversión demasiado alta para la mayoría de departamentos responsables de la iluminación}

El costo inicial de luminarias LED por todos los elementos de su fabricación es sumamente elevado comparado con luminarias de tecnología tradicional. Por ejemplo, una luminaria de sodio de alta presión de 250 vatios puede costar en el mercado ecuatoriano \$200 dólares mientras que una luminaria LED de 80 vatios podría costar alrededor de \$1,200 dólares. La implementación de iluminación vial LED por grandes contratos es algo que muchas empresas no pueden cubrir por su elevado costo inicial.

Si se cubren los costos iniciales se deben descuidar otras áreas debido a los limitados presupuestos, una decisión de inversión en iluminación LED masiva significaría baja inversión en alcantarillado, carreteras u otras necesidades de la población.

\section{Comentarios finales}

El consumo energético mediante iluminación LED es reducido considerablemente y con ello el gasto económico en energía eléctrica de los respectivos entes responsables.

Previo a la implementación masiva de luminarias LED, se debe considerar las opciones de tecnología que ofrece el mercado, sus costos iniciales, los de explotación y mantenimiento del sistema de iluminación, 
así como los costos directos e indirectos consecuencia de iluminar un área.

Una afectación a la fauna, tendría sus consecuencias en el impacto sobre el flujo de turismo, afectando a su vez la actividades económicas que se desarrollan gracias a este, por lo que es necesario evaluar las consecuencias de iluminar un sector, en especial en lugares con ecosistemas frágiles.

El cumplimiento de las demandas visuales de los usuarios de iluminación exterior, en ocasiones debe considerar el empleo de fuentes de luz cuyo espectro de descarga se encuentre enriquecido en los azules, sin embargo su aplicación debe realizarse considerando las afectaciones tanto positivas como negativas del lugar a iluminar. No existe una única recomendación para la transición de tecnología, la incursión en tecnología led deberá ser evaluada y revisada por su contexto, dado que no resulta equivalente iluminar un área geográfica con alta biodiversidad que una metrópoli.

\section{Referencias}

Boyce, P. R. (2009). Lighting for Driving. Roads, Vehicles, Signs, and Signals. Boca Raton, F1: Taylor \& Francis Group.

DiLaura, D. L., Houser, K. W., Mistrick, R. G., \& Steffy, G. R. (2011). The Lighting Handbook. New York: Illuminating Engineering Society of North America.

Elvidge, C. D., Keith, D. M., Tuttle, B. T., \& Baugh, K. E. (2010). Spectral Identification of Lighting Type and Character. Sensors, 10(4), 3961-3988.

Flesch, P. (2006). Light and Light Sources. High Intensity Discharge Lamps. Berlin: Springer.

Hecht, J. (2015). Environment, Wlidlife and Led Illumination. Optics \& Photonics News, 26(11), 42-47. 
Medina, J. C., Avrenli, K. A., \& Benekohal, R. F. (2013). Field and Software Evaluation of Illuminance from LED Luminaires for Roadway Applications. Transportation Research Record: Journal of the Transportation Research Board, 55-64.

Minicomercio Industria y Turismo. (21 de 09 de 2016). Informes de turismo. Obtenido de Centro de Información Turística - CITUR: http://www.mincit.gov.co/publicaciones.php?id=16590 Ministerio de Turismo. (30 de diciembre de 2014). Resumen 2014: Turismo ecuatoriano, en su mejor momento. Recuperado el 12 de 10 de 2016, de http://www.turismo.gob.ec/resumen-2014-turismo-ecuatoriano-en-su-mejor-momento/

Osram A. (2016). High-pressure sodium vapor lamps for open and enclosed luminaires. Recuperado el 12 de 10 de 2016, de https://www.osram.com/osram_com/products/lamps/ high-intensity-discharge-lamps/high-pressure-sodium-vapor-lamps-for-open-and-enclosed-luminaires/index.jsp

Osram b. (26 de junio de 2012). LED Fundamentals. Driving LEDs AC-DC Power Supplies.

Pelka, D., \& Patel, K. (2003). An overview of LED applications for general illumination. SPIE Proceedings, Design of Efficient Illumination Systems (págs. 15-26). San Diego: SPIE Digital Library.

Phillips a. (2016). Luminarias público y residencial. Obtenido de http://www.lighting.philips.es/prof/luminarias-de-exterior/ alumbrado-publico-y-residencial/luminarias-publico-y-residencial\#pfpath=0-OCOUTD_GR-NROAD_CA

Phillips b. (2016). SON Sodio Alta Presión. Obtenido de http:// www.lighting.philips.es/prof/lamparas-profesionales/ lamp.-de-descarga-alta-intensidad/son-sodio-alta-presion\#pfpath=0-EP01_GR-EP01LHID_CA 
Rich, C., \& Longcore, T. (2006). Ecological Consequences of Artificial Night Lighting. Washington: Island Press.

Valentová, M., Quicheron, M., \& Bertoldi, P. (2014). LED Projects and Economic Test Cases in Europe. Joint Research Centre, Institute for Energy and Transport. Ispra: Joint Research Centre of the European Commission. 